\title{
Assessment of mechanical properties of compression socks using cut-strip method
}

\begin{abstract}
The aim of this research was to analyze mechanical behavior of compression pressure using cut-strips at ankle portion. For mechanical characterization, cut-strip samples were analyzed for their interaction to constant rate of loading and un-loading, cyclic loading upto 20 cycles and dwell time effect ( 3 minute) on tensile decay suing tensile tester (Testometric M350-5CT). All of the results were statistically analyzed by Minitab software using simple linear regression analysis ( $\mathrm{p}$ value $<0.005$ ) and co-relationship values.
\end{abstract}

Keywords: compression pressure (mmHg), Extension (mm), cyclic loading and unloading Vs extension, Laplace's law, dwell time, force-elongation curve

\author{
Volume 5 Issue 5 - 2019
}

\author{
Hafiz Faisal Siddique \\ Clothing technology, Technical University of Liberec, Czech \\ Republic
}

Correspondence: Hafiz Faisal Siddique, Clothing technology,
Technical University of Liberec, Liberec, Czech Republic, Tel +420775321349, Email faisalsidique3648@tul.cz

Received: August 23, 2019 | Published: September 09, 2019

\section{Introduction}

Compression socks are the highly recommended compression garment for pressure exertion on the lower part of the leg. It is used to reduce venous hyper pressure.

Mechanism of action is lowering of pressure exertion from ankle to calf portion of the leg. The pressure exertion value should be highest at the ankle that must gradually decrease along the direction of the leg. This varying degree of compression pressure propagate and regulate blood flow, keep the muscles in-line at the right position to mitigate the injury risk, gives relief to many of chronic venous disease patients and used for therapeutic purposes. ${ }^{2,3}$

Mechanical properties (stiffness Vs friction and elasticity/ extensibility Vs hysteresis) of compression stockings correlate with intensity of deliverance of pressure, durability, comfortability and medical efficacy in compression therapy. These mechanical features are mainly influenced by nature of material, density of fabric, fabrication technology and delivery modes. Compression socks compatibility to human body (physical-mechanical interaction) is of great importance and is based on static and dynamic working mechanism explained by Laplace's law and Pascal's law. Static compression (no motion of leg/ patient) deliverance is based on Laplace's law while that of dynamic compression (patient/leg in motion) based on Pascal's law. ${ }^{4}$

Rong Liu et al. ${ }^{5}$ studied mechanical (tensile, bending and shearing) and surface properties of different socks samples comprised of different compositions, thickness and GSM in both wales and course direction using Kawabata Standard Evaluation System. But surprised how he did as the socks area is not much and easy to install on KAWABATA device. Anyhow, he concludes that; Tensile indices like (WT, EM) contribute strongly and exhibit strong co relationship with compression pressure While, shearing $(\mathrm{G}, 2 \mathrm{HG})$, bending $(\mathrm{B})$ and weight (W) properties exhibit medium but positive linear correlations with skin pressure gradient distribution. ${ }^{5}$

Hugo Partsch et al. ${ }^{6}$ compared interface pressure and stiffness properties of compression socks. He used cut-strips and tested for stretch tension curves using Zwick dynamometer. They concluded that interface pressure values measured by the MST tester at B1 are comparable with the values calculated from the force/extension-curves obtained using dynamometer. But the gap observed in his research that he has not mentioned that as he washed the socks samples are not, it is also not clear at what extension levels he extended the cut-strips whether equal to circumference of the leg at ankle portion or some fixed stretch percentage. Anyhow, he concluded that correlation was highly significant, $\mathrm{P}<.0001 ; \mathrm{r}=0.8161){ }^{6}$

Harpa et al. ${ }^{2}$ studied the tensile and rheological features of compression socks before use, after 15 and 30 wearing-washing cycles in wales direction using Mesdan Tensolab. He proposed Grab test method as real simulator of durability assessment of compression socks. He concluded that the proposed testing (Grab test) method can be used to assess the graduated compression socks by measuring the deformation resulting in traction tests in three directions under two different levels of loads $\left(\mathrm{F}_{\min } 0.65 \mathrm{~N}\right.$ and $\left.\mathrm{F}_{\max } 1.2 \mathrm{~N}\right)$ but still gap he could not fill about hand washing of socks samples or cut strips, why he selected $100 \mathrm{~mm} / \mathrm{mint}$ rate of extension in his study. ${ }^{2}$

Lijing Wang et al. ${ }^{7}$ studied the mechanical properties of compression socks. For this he measured tensile properties as per Australian Standard (AS) 2001.2.3.1-2001, stretch-recovery tests as per BS EN 14704-1:2005 standard while bursting strength using AS 2001.2.19. They concluded that compression fabrics are strong with a breaking load greater than $200 \mathrm{~N}$ have excellent stretchability with breaking extension well beyond $200 \%$ in both wale and course directions. After fatigue stretching, the average immediate recovery of compression fabric is more than $95 \%$ and the average elastic recovery after an extended period of relaxation (1-24 hours) is at least $98 \%$. After 3 weeks service and a few hours relaxation, the compression fabric has only around $2 \%$ residual. $^{7}$

Homa et al. ${ }^{8}$ investigated the pressure change using weft knitted fabrics over time. For this, plain and interlock fabrics of different stitch lengths at different strains were tested for interfacial pressure before and after each 48 hours. The same tests were performed after repeated washing and repeated usage. She concluded that stitch length is a significant factor affects pressure values and pressure reduction percentages over a prolonged time (48 hours). In plain fabric, lowest stitch length declines more than interlock fabric. In the both constructions, extension significantly influences interfacial pressure. ${ }^{8}$ 
Yongrong Wang et al. ${ }^{9}$ studied dynamic pressure reduction of elastic fabric as it exhibit hysteresis and pressure decay during wearing at varying extension levels $10 \%-40 \%$. He introduced a new method to measure garments pressure. The pressure measuring device is composed of a rigid hemisphere contains three pressure sensors and a sample-holding device calculated using below equation.

Dynamic pressure reduction=Dynamic pressure at $1^{\text {st }}$ cycle Dynamic pressure at $5^{\text {th }}$ cycle

The sensors sense static and dynamic pressure as hemisphere goes down and then up at predetermined press depths, velocities and test cycles under control of the displacement driving device; the sample deforms to some extent and produces pressure on the surface of hemisphere. He has concluded that plain fabrics have higher dynamic pressure at all the extensions $(10 \%-40 \%)$ and is reduced with the repeated extension $(10 \%-40 \%)$ and recovery. ${ }^{9}$

The intensity of compression pressure used for various diseases is categorized as moderate up to $(20-30 \mathrm{mmHg})$ and firm compression $(30-40 \mathrm{mmHg})$. This extent of pressure is decided and recommended to treat circulatory and vascular medical conditions as well for tired, sore, swollen, or aching legs. ${ }^{9-12}$

The aim of this research was to evaluate the mechanical behavior of compression socks including Loading-unloading Vs extension followed by cyclic loading effect on tensile decay after 20 cycles, 3 minutes dwell time effect at various extension of hand washed socks samples that is not commonly followed by most of authors that can attribute to more accurate results for the verification of efficiency of compression socks. On the basis of these cut strips mechanical results we can judge the performance of compression socks under different dynamic as well as static loads.

\section{Materials and methods}

Three type of compression socks were purchased and structurally analyzed at the ankle portion with great precision and accuracy. Here, we selected and compared 2 socks samples (single jersey and Rib knit) of same compression level, Class II and one socks sample of compression class III (Single Jersey) compared with compression class II (single jersey) as shown in below given flow chart (Figure 1). These custom-made socks samples readily available for patients were analyzed rather to develop.

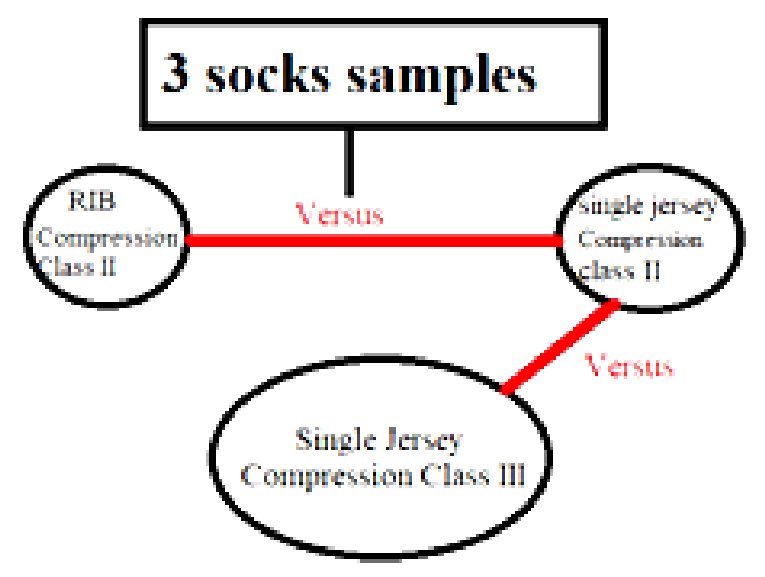

Figure I Flow chart of socks comparison .

\section{Physical testing of compression socks}

All the socks samples were evaluated for stitch densities, thickness and areal densities in relaxed state under controlled atmospheric condition, (RH, $65 \pm 5 \%$, temperature, $20 \pm 2^{\circ} \mathrm{C}$ ). Results are given in Table 1.

\section{Marking and cutting of compression socks}

Firstly, we put-on all the socks samples on leg and marked a line at $12 \mathrm{~cm}$ height from the floor as a mean line and then unworn the socks and keep flat on table for relaxation. After relaxation, we marked a square line of dimension $\left(5 \times 5 \mathrm{~cm}^{2}\right)$ on face of each compression socks samples named as BIISJ, BIIISJ and DGIIRIB as shown in Figure 2. Here, B and DG stands for beige and dark grey color while II and III as compression levels while SJ and RIB stands for single jersey and rib knit types. All the three pairs of socks samples were worn on wooden leg (Circumference: $23 \mathrm{~cm}$ ) simultaneously and tested for dimensional change and compression pressure measurement. Each pair of socks was cut to strips of each dimension $(8 \times 5 \mathrm{~cm})$ simultaneously to hang on tensile testing machine.

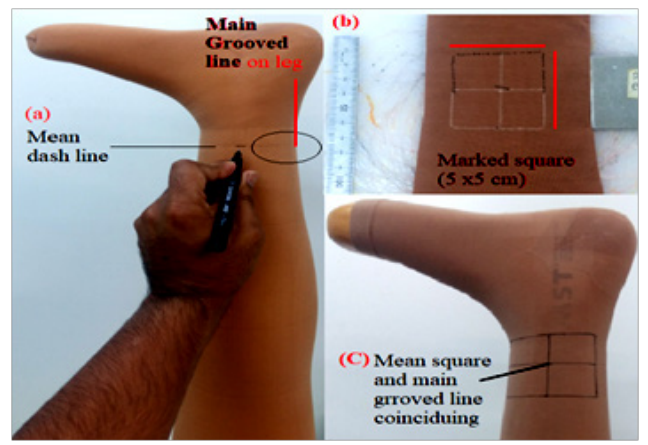

Figure 2 Marking on face of socks.

\section{Washing procedure}

Before testing for tensile characterization, all socks cut-strip samples were gentle detergent based hand washed. Washing was performed by dipping all the socks samples in solution of 4 liters of normal water and $8 \mathrm{gm}$ of ECE detergent (non phosphorous and without Optical brightening agent) and mixed thoroughly as per Australian guidelines AS-2001:5-2005. As per standard, all samples were flat dries by placing all the pairs of socks samples between layers of towels as shown below Figure 3 for 24 hours under standard atmospheric condition $\left(\mathrm{RH}, 65 \pm 5 \%\right.$, temperature, $\left.20 \pm 2^{\circ} \mathrm{C}\right) .{ }^{13}$

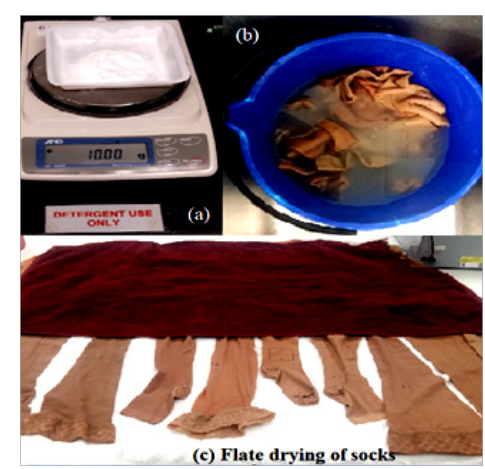

Figure 3 Hand washing of compression socks. 


\section{Mechanical testing of compression socks strips}

Three cut-strip samples were installed on tensile testing machine (Testometric M350-5CT) vertically following European standard test method EN ISO-1934-1:1992. Strips were stretched transversely similar to circumferential direction of wooden leg. Tensile testing machine was used to measure the different tensile properties. Stretching machine is comprised of two jaws; one was fixed while other was moveable under load cell of $100 \mathrm{~N}$, gauge length of $50 \mathrm{~mm}$, at constant rate of elongation of $500 \mathrm{~mm} / \mathrm{min}$.

Here, strip samples were extended to $40 \mathrm{~mm}$ compared to gauge length $(50 \mathrm{~mm})$. Total length $(90 \mathrm{~mm})$ was sum of gauge length $(50 \mathrm{~mm})$ and extended length $(40 \mathrm{~mm})$. The values of extended force comparison to circumference of the leg were calculated using formula of stretch percentage given below Figure 4. ${ }^{5,12,14,15}$

$$
S \%=\frac{L c-S c}{S c} \times 100
$$

$L c=$ Leg circumference

$S c=$ Socks circumference
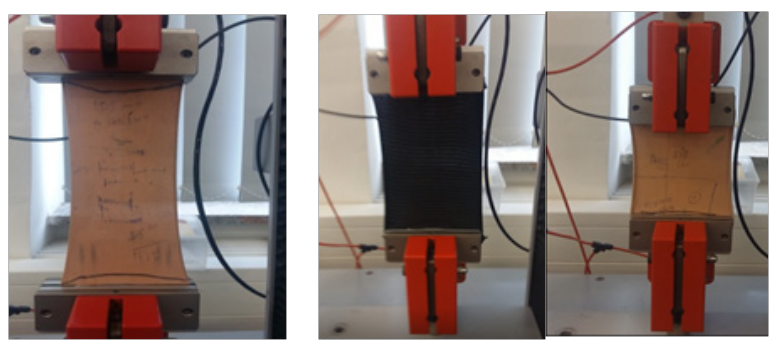

Figure 4 Measurement of tensile characteristic of cut-strip sample.

For testing three types of sample strips, machine adjustments were almost fixed. All the three socks strip samples were tested for Loading-unloading Vs extension relationship, cyclic loading and unloading effect on tensile decay while effect of dwell time on tensile decay. After these tests, tensile values at specific extensions were compared and analyzed statistically.

\section{Results and discussion}

\section{Loading-unloading Vs extension effect}

Figure 4 portrays that BIISJ socks sample exhibits lowest value of tensile force when was extended to maximum $40 \mathrm{~mm}$ (50\%) compared to other socks sample (DGIIRIB and BIIISJ) as shown in graph 1. While socks sample BIIISJ acquired highest tensile force to be extended upto $50 \%$.

We also found from the Figure 5, strip sample BIISJ exhibit least structural deformation (red lines) followed by DGIIRIB (blue lines). Strip sample BIIISJ (green lines) exhibit high value of deformation when relaxed to no extension as shown in Figure 5. The reason of such a behavior of all the cut-strips is due to varying gram per square meter (gsm values) from $308.80,350.97$ and 378.47 of BIISJ, DGIIIRIB and BIIISJ respectively. Another reason of such a behavior cut-strips is due to varying linear densities of inlaid thread elastane yarn thread values ranges from $310 \mathrm{D}, 360 \mathrm{D}$ and $520 \mathrm{D}$ respectively as given in Table 1. The data for loading-unloading Vs extension was analyzed statistically by Minitab 17 software using regression analysis tool. The data of statistical analysis is given below in Table 2. Here, we can claim that, as extension increases there is a significant ( $p$ value $<0.05$ ) increase in tensile properties of each three strip samples and vice versa as given in Table 2 .

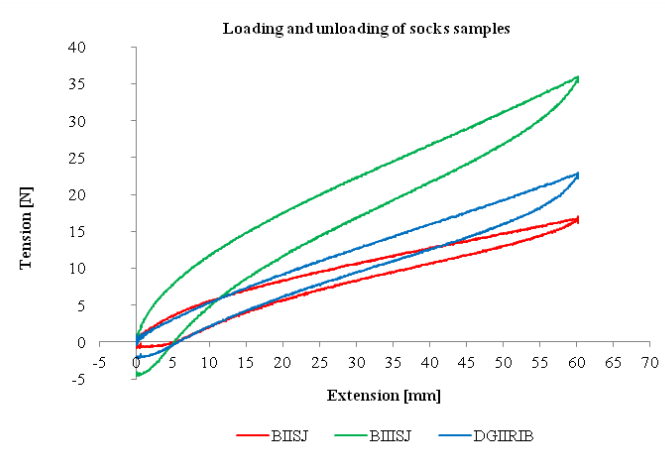

Figure $\mathbf{5}$ Loading stress to extend compression.

Table I Physical measurement of compression socks

Sample codes

BIISJ* BIIIS]* DGIIRIB*
Socks Circumference

(cm)

Courses/inch

Wales/inch

Stitch Density (Stitch/ inch2)

Thickness b, (mm)

Areal Density (GSM)

Linear density of elastane inlaid thread (denier)

Type of inlaid yarn

Type of Main yarn

Fiber Analysis (\%)
$16.6 \quad 15.4$

57

49

52

49

42

460

$0.75 \quad 0.95$

308.8

378.47

350.97
*B, beige; II, III, compression class; SJ, single jersey; RIB, rib structured; PA, polyamide 
Table 2 Statistical analyzed results

\begin{tabular}{|c|c|c|c|c|c|}
\hline Parameters & Socks samples & R-Square value (\%) & $p$ value $<0.05$ & Co-relation & Regression equation \\
\hline Loading stress & BIISJ & 97.93 & 0.001 & 0.99 & $Y=2.928+0.2409 X$ \\
\hline \multirow{2}{*}{$\begin{array}{l}\text { Vs extension } \\
\text { effect }\end{array}$} & BIIISJ & 98.48 & 0.001 & 0.99 & $Y=6.218+0.5090 X$ \\
\hline & DGIIIRIB & 99.66 & 0.001 & I & $Y=1.708+0.3550 X$ \\
\hline \multirow{3}{*}{$\begin{array}{l}\text { Un-loading } \\
\text { strain Vs } \\
\text { detraction } \\
\text { effect }\end{array}$} & BIISJ & 99.27 & 0.001 & 1 & $Y=-0.536+0.2794 X$ \\
\hline & BIIISJ & 98.61 & 0.001 & 0.99 & $Y=-2.535+0.6131 X$ \\
\hline & DGIIIRIB & 99.49 & 0.001 & I & $Y=-I .80 I+0.3675 X$ \\
\hline
\end{tabular}

\section{Cyclic loading and unloading effect on tensile decay}

Similarly, three socks strips were clamped in transversal direction of socks. We extended all the three socks strip samples upto $40 \mathrm{~mm}$ ( $50 \%$ extension) reference to original length (gauge length $=50 \mathrm{~mm}$ ) simultaneously. After $50 \%$ extension upto $90 \mathrm{~mm}$ of extension, we allowed the strip samples to detract for $0.5 \mathrm{~cm}(5 \mathrm{~mm})$ upto $35 \mathrm{~mm}$ of extension from $40 \mathrm{~mm}$ of maximum extension. We continued the loading and unloading in to and fro motion for $5 \mathrm{~mm}$ [ $40 \mathrm{~mm}$ to $35 \mathrm{~mm}$ then to $40 \mathrm{~mm}$ ] till 20 cycles and found an incredible decay in tensile properties while loading and unloading after 20 cycles as shown in Figure 6 and Figure 7.

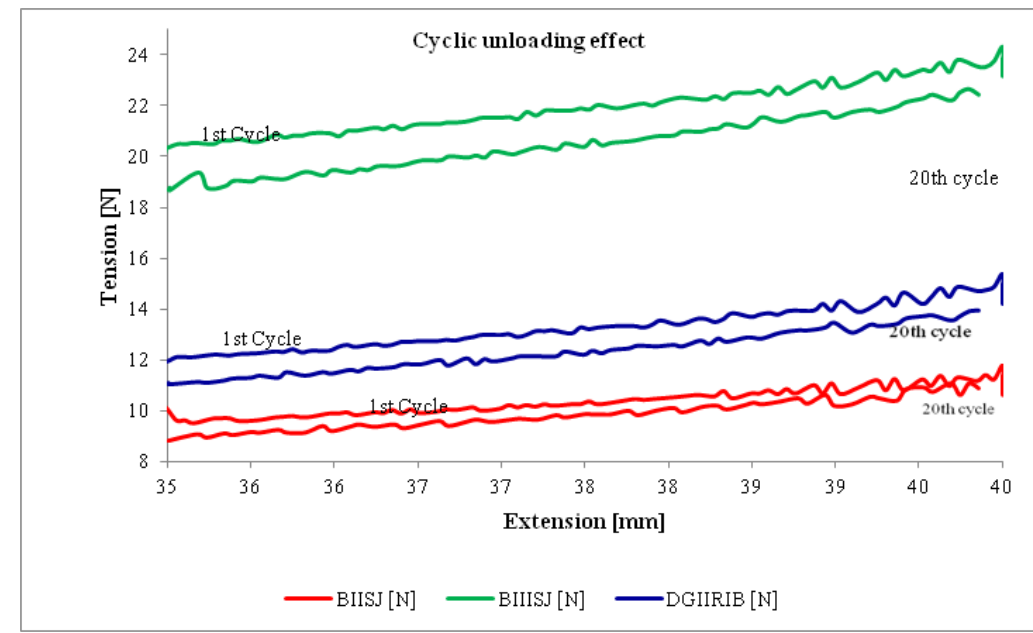

Figure 6 Cyclic unloading effects on tensile decay.

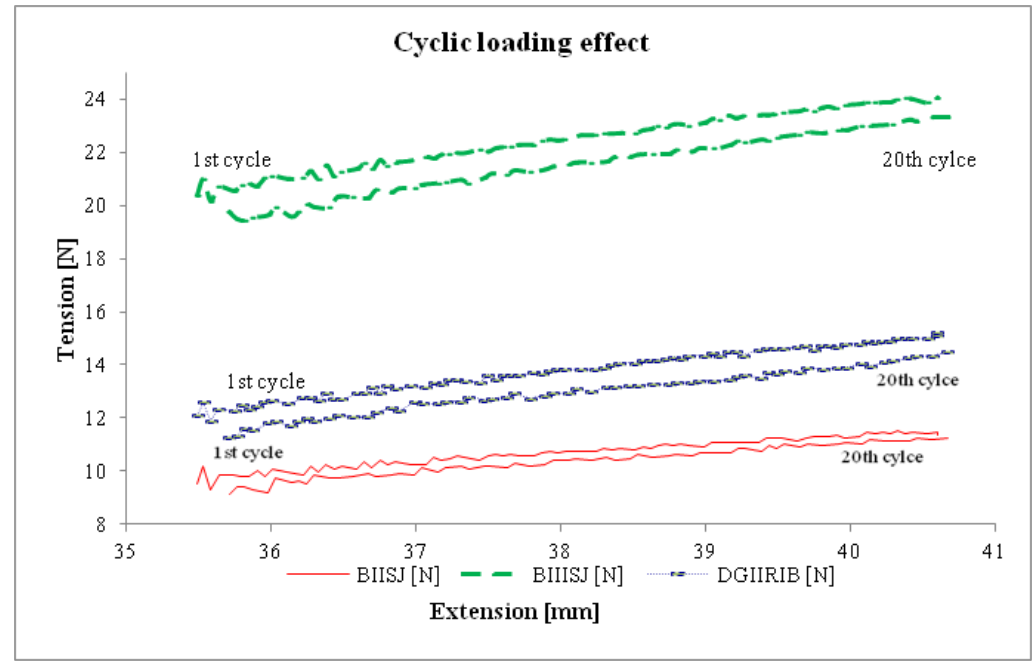

Figure 7 Cyclic loading effects on tensile decay. 
Figure 6 and Figure 7 portray that all the three strip samples BIISJ, DGIIRIB and BIIISJ decayed their tensile value as the number of loading and unloading cycle increases.

We observed from the Figure 6 and Figure 7 that at $1^{\text {st }}$ cyclic loading, tensile force was higher against each socks strip samples BIISJ, DGIIRIB and BIIISJ which was declined to lower value with increasing number of cycles. At 20 cycles, there was a significant decline due to fragility of elastane thread double covered by polyamide multifilament.
To and fro cyclic loading-unloading was analyzed statistically by Minitab 17 software using regression analysis tool. The data of statistical analysis is given below in Table 3. Here, we can claim that, as loading and unloading of socks strip samples continued to numerous cycles (up to 20 cycles), the decayed their tensile force due to permanent structural deformation of elastic materials. This cyclic loading and unloading has a significant impact ( $\mathrm{p}$ value $<0.05$ ) on tensile decay values of socks strip samples.

Table 3 Statistical analysis of each parameter

\begin{tabular}{|c|c|c|c|c|c|}
\hline Parameters & Socks samples & R-Square value (\%) & p value $<0.05$ & Co-relation & Regression equation \\
\hline \multirow{6}{*}{$\begin{array}{l}\text { Cyclic Loading } \\
\text { effect on tensile } \\
\text { decay }\end{array}$} & BIISJ (I ${ }^{\text {st }}$ Cycle) & 95.18 & 0.001 & 0.96 & $Y=9.782+0.0222 X$ \\
\hline & BIISJ (20 Cycle) & 97.44 & 0.001 & 0.98 & $Y=9.358+0.0313 X$ \\
\hline & DGIIRIB (I ${ }^{\text {st }}$ Cycle) & 98.63 & 0.001 & 0.98 & $Y=12.278+0.0362 X$ \\
\hline & DGIIRIB (20 $0^{\text {th }}$ Cycle) & 98.24 & 0.001 & 0.99 & $Y=11.53 \mid+0.045 X$ \\
\hline & BIIISJ (I Ist Cycle) & 98.42 & 0.001 & 0.99 & $Y=20.616+0.0451 X$ \\
\hline & BIIISJ (20 Cycle) & 99.47 & 0.001 & 0.99 & $Y=19.503+0.0629 X$ \\
\hline \multirow{6}{*}{$\begin{array}{l}\text { Cyclic Un- } \\
\text { loading effect } \\
\text { on tensile decay }\end{array}$} & 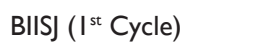 & 95.35 & 0.001 & 0.98 & $Y=-2.3188+0.3383 X$ \\
\hline & BIISJ (20 th Cycle) & 97.22 & 0.001 & 0.99 & $Y=-4.874+0.3934 X$ \\
\hline & DGIIRIB (I ${ }^{\text {st }}$ Cycle) & 98.59 & 0.001 & 0.99 & $Y=-7.1918+0.5463 X$ \\
\hline & DGIIRIB (20 th Cycle) & 98.18 & 0.001 & 0.99 & $Y=-9.0044+0.5701 X$ \\
\hline & BIIISJ (I Ist Cycle) & 98.35 & 0.001 & 0.99 & $Y=-3.2167+0.671 X$ \\
\hline & BIIISJ (20 ${ }^{\text {th }}$ Cycle) & 99 & 0.001 & 0.99 & $Y=-8.3972+0.7724 X$ \\
\hline
\end{tabular}

\section{Effect of dwell time on tensile decay}

All the three strips were clamped simultaneously and extended to three different extensions, $10 \mathrm{~mm}, 20 \mathrm{~mm}$ and $30 \mathrm{~mm}$. At each extensions level, we dwelled the socks samples and observed the decline in tensile decay occurred with same rate after extension as shown in Figure 8.

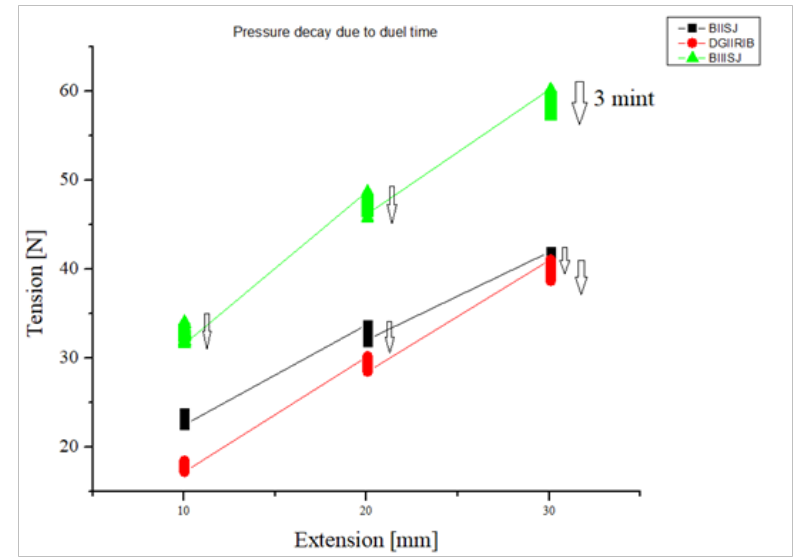

Figure 8 Dwell time effect on tensile decay.

Figure 8 portray that as clamped socks extended to $10 \mathrm{~mm}$ followed by $20 \mathrm{~mm}$ and $30 \mathrm{~mm}$, we found that after each extension there is incredible increase in tensile force of all the three socks samples accordingly their stiffness. It is also observed that when the extension of socks is stopped and allowed to recover for 3 minutes, a significant decrease in tensile force is observed highlighted with arrows.

\section{Conclusion}

In this research we tried to find out the durability measurement of compression socks. How the behavior of socks exploited when exposed to different mechanical impacts. This direct or indirect exploitation of compression socks mitigate the efficacy as well as prolonged performance of compression socks.

We have concluded that when compression socks are extended similar to circumference of leg, it continued to stretch and when relaxed to original length (no extension), there observed some structural deformation. We also extended the socks for 20 cycles for $\pm 5 \mathrm{~mm}$ loading and unloading simulating the contraction and relaxation of muscles and concluded that tensile values declined ultimately reducing performance and efficacy of compression socks. We also concluded that when socks is extended to some specific level $10 \mathrm{~mm}, 20 \mathrm{~mm}$ and $30 \mathrm{~mm}$ along and were dwelled at each step for even short period of time (3mint), there observed a significant decline in tensile values which reflects how much compression pressure decrease after short span of time.

\section{Acknowledgments}

This work was supported by the technical University of Liberec, Czech Republic under the project of under the project of Student Grant Scheme SGS-19. Project reference number is 21309. 


\section{Funding}

None.

\section{Conflicts of interest}

The authors declare that they have no competing interests.

\section{References}

1. Flaud P, Bassez S, Counord J. Comparative in vitro study of three interface pressure sensors used to evaluate medical compression hosiery. Dermatol Surg. 2010;36(12):1930-1940.

2. Harpa R, Piroi C, Doru Radu C. A new approach for testing medical stockings. Textile Research Journal. 2010;80(8):683-695.

3. Smith MWL, Dalbey JC. Gradient compression hosiery knitted using core spun yarns. 7895863 B2, USA; 2010.

4. Hugo P. The use of pressure change on standing as a surrogate measure of the stiffness of a compression bandage. Eur J Vasc Endovasc Surg. 2005,30(4):415-421.

5. Rong L, Yi Lin K, Yi L, et al. Objective evaluation of skin pressure distribution of graduated elastic compression stockings. Dermatol Surg. 2005;31(6):615-624.

6. Hugo $\mathrm{P}$, Bernhard $\mathrm{P}$, Walter $\mathrm{B}$. Interface pressure and stiffness of readymade compression stockings: Comparison of in vivo and in vitro measurements. J Vasc Surg. 2006;44(4):809-814.
7. Wang L, Felder M, Cai Y J. Study of properties of medical compression garment fabric. Journal of Fiber Bioengineering and Informatics. 2011;4(1):15-22.

8. Homa M, Marzie A, Sadeghi AH, et al. On the pressure behavior of tubular weft knitted fabrics constructed from textured polyester yarns. Journal of Engineered Fibers and Fabrics. 2011;6(2):30-39.

9. Yongrong W, Peihua Z, Yiping Z. Experimental investigation the dynamic pressure attenuation of elastic fabric for compression garment. Textile Research Journal. 2014;84(6):572-582.

10. Sau-fun N, Chi-leung H, Lai-fan W. Development of medical garments and apparel for the elderly and the disabled. 2011;43(4):235-285.

11. Macintyre L, Baird M, Weedall P. The study of pressure delivery for hypertrophic scar treatment. International Journal of Clothing Science and Technology. 2004;16(1/2):173-183.

12. Ng SF, Hui CL. Pressure model of elastic fabric for producing pressure garments. Textile Research Journal. 2001;71(3):275-279.

13. ASTM D4964. Standard test method for tension and elongation of elastic fabrics (constant-rate-of-extension type tensile testing machine). 2016

14. Ng SF, Hui CL. Pressure model of elastic fabric for producing pressure Textile Research Journal. 2001;71(3):275-279.

15. RAL-GZG 387. Medical compression hosiery. Quality and Labeling, German Institute for Insurance and Marking, Germany: E.V. German Institute for Health; 2008. 